Article

\title{
Searching for Supersymmetry at LHC Using the Complex-Network-Based Method of the Three-Dimensional Visibility-Graph
}

\author{
Susmita Bhaduri *(-) and Anirban Bhaduri $(0)$ \\ Moonshot Analytics and Design, 3/1/1 Dhakuria Station Lane, Kolkata 700031, India; \\ bhaduri.anirban@moonshot.net.in \\ * Correspondence: susmita.sbhaduri@dgfoundation.in; Tel.: +91-983-613-2200
}

Received: 17 August 2020; Accepted: 2 September 2020; Published: 10 September 2020

check for updates

\begin{abstract}
For the last several decades, there has been tremendous interest in search for Supersymmetry (SUSY) in the area of high energy physics. At Large Hadron Collider (LHC), there have been continuous searches for SUSY for prompt and non-prompt, for particle $R$-parity conserving and $R$-parity violating generation and decays. The limits obtained from these experiments and analyses for detection of the signatures of supersymmetric particles (LSP), revealed greater possibilities of such experiments in the collider. However, these signatures are usually derived under the assumption of bit optimistic conditions of the decaying process of sparticles to the final states. Moreover, SUSY might have been in a disguised state at lower mass-scales as a result of difficult and challenging mass spectra and mixed modes of decays. In this investigation, a novel method of 3-dimensional (3D) Visibility-Graph Analysis is proposed. This is an extension of Visibility Graph analysis of data series to perform the scaling analysis for 3D space. The experimental data spaces analyzed are made up of the component-space (in the $X, Y$ and $Z$ coordinates) of transverse momentum $\left(p_{T}\right)$ values taken out from 4-momenta of the signatures of the final state of the pair of mega-jets extracted from the multiJet primary $p p$ collision data from Run B of 2010 at $7 \mathrm{TeV}$ which was used for the search of SUSY using razor filter. The symmetry scaling and the inherent scaling behavior, scale-freeness of multi-particle production process is studied in terms of 3D Power-of-Scale-freeness-of-Visibility-Graph (3D-PSVG) extracted from the 3D Visibility Graphs constructed out of the experimental data spaces. The signature of SUSY may be identified by analyzing the scaling behavior and long-range correlation inherent in the 3D space made up of signatures of final state of multi-particles produced in the $p p$ collision at $7 \mathrm{TeV}$, for the analysis of SUSY, which the conventional method of analyzing the spectrum of invariant mass or $p_{T}$ may miss.
\end{abstract}

Keywords: Supersymmetry; complex network; 3D Visibility-Graph Analysis; symmetry-based scaling; transverse momentum

PACS: 24.60.Ky; 24.60.Lz; 29.90.+r; 11.30.Pb; 64.60.al; 89.75.-k

\section{Introduction}

\author{
nāsadāsīnno sadāsittadānīm \\ nāsidrajo no vyomā paro yat \\ kimāvarīvah kuha kasya sármann \\ ambhah kimāsìd gahanam gabhïram \\ tama āsīttamasā gūụhamagre \\ apraketam salilam sarvamā idam
}


tucchyenābhvapihitam yadāsīt

tapasastan-mahinājāyataikam

"THEN" was not non-existent nor existent:there was no realm of air, no sky beyond it. What was covered in, and where? And what gave shelter? Was water there, unfathomed depth of water? Darkness there was: at first concealed in darknew (new darkness) this all was indiscriminate chaos. All that existed then was void and formless:by the great power of warmth was born that Unit.

\section{Rig-Veda 10.129.1,3 -Translated by R.T. Grffith.}

Over the past few decades, the Standard Model (SM) of particle physics has been giving us an elaboration of the fundamental particles and their respective interactions [1-3]. UA1 and UA2 experiments in 1983 have confirmed that for short-range weak interactions the $W$ and $Z$ bosons have huge mass but bosons in SM have to be massless to keep SM Lagrangian invariant under local gauge transformations. Brout, Englert, Higgs mechanism suggested a probable solution where gauge bosons can gain mass in gauge invariant process through spontaneous symmetry breaking [4-6]. Glashow, Weinberg and Salam have integrated this concept with the SM by formulating the theory of $W$ and $Z$ bosons with huge mass and an additional elementary 0-spin particle which is the Higgs boson interacting with the gauge bosons and the fermions. The discovery of the Higgs boson by ATLAS [7] and CMS [8] Collaborations at the LHC [9] gave final approval to the SM. The conjectures of the SM have been established experimentally with increasing robustness.

However, SM has a few challenges. For example, gravitational interactions cannot be included in this model. The origination of charge parity violation yielding to matter versus antimatter and also dark matter is not identified in this model. It also does not answer how neutrinos gain mass and so on. There has been search for a framework which unifies all interactions. For resolution of the SM limitations, many theories have come up. Among them, supersymmetry (SUSY) has been one of the most reasonable extensions of the SM in the field of particle physics [10-17]. It allows us to determine the coupling unification at the energy of $10^{16} \mathrm{GeV}$, and emerges as a prospective candidate for dark matter-lightest supersymmetric particle (LSP) [18] which is a mandatory element for decoding the concept of quantum gravity based on the framework of string theory. The signature of SUSY in the detector is driven by the nature of the LSP which is dependent on the underlying process of SUSY breaking. The SUSY model eliminates the quadratic divergences in radiative modifications of the Higgs boson mass by default. For each particle in the SM, SUSY infuses a super-partner or the sparticle, with a spin difference from the SM particle by $1 / 2$ unit. There exists theoretical propositions which suggest that the masses of sparticles might be less than $\sim 1 \mathrm{TeV}[16,17]$ and hence the experiments at the LHC are best suited to be analyzed for the discovery of SUSY particles. For the past decades, a considerable amount of missing transverse energy has been considered as the most rigorous observation for the identification of the production and decay of SUSY particles at colliders and the quantity of missing transverse energy depends on the division of mass among the heavier sparticles. To date, conventional SUSY searches in CMS $[19,20]$ needed several jets with large $p_{T}$ correlated with large values of $H_{T}$, i.e. the scalar sum of the transverse momenta of all the jets satisfying the jet selection criteria, and absence of large transverse energy to distinguish a SUSY signal from the very large SM backgrounds. In the work [21], comparatively moderate criteria of absence of transverse energy (i.e. $\left.\not Z_{t}\right)\left(Z_{t}>40 \mathrm{GeV}\right)$ and also $H_{T}\left(H_{T}>120 \mathrm{GeV}\right)$, have been used. The SM has been extended with softly broken SUSY [18,22-25] to predict new elementary particles which are a super-partner of the SM particles. The Fermilab Tevatron [26,27] and the CERN LHC (e.g., [28-30]) have concentrated on the events having signatures of high-energy hadronic jets and leptons decayed from squarks and gluinos produced in pairs, under the assumption of $R$-parity [31] conservation. These events usually have a high degree of missing transverse energy resulting from the weakly interacting super-partners which are stable. One of the super-partners is generated in each of the two decay chains. In these final states, searches for SUSY were conducted by the ATLAS [32] at $13 \mathrm{TeV}$ and CMS [33] Collaborations at $8 \mathrm{TeV}$. CMS [34,35] have applied the razor approach to search for LSP, after considering the $R$-parity [31] conservation.In this 
work they have considered two analyses-a comprehensive search for new heavy-particle-pairs which decay to final states with at least two jets and missing transverse energy and an exclusive search for the final states having at least one jet arising from a bottom quark. For both of the analyses the final states of hadronic, single-lepton and di-lepton events from the multiJet primary dataset of the Run B (2010) of $p p$ collision at $7 \mathrm{TeV}$ from CMS Collaboration [36] are considered. CMS [34,35] have analyzed chosen events in the two-dimensional razor plane of $M_{R}$-razor kinematic variable, defined with respect to the momentum of the two mega-jets and $R$, a variable without dimensions signifying the missing transverse energy. These razor variables are established on the generic procedure of the production of two heavy particles in pair, each one decaying to an unidentified particle with visible decay products. The two-dimensional shape analysis in the razor-variable plane is validated with simulated events, and no remarkable excess over the background expectations has been noticed. The output of the search for SUSY [34,35] has been used to drive simplified SUSY models in [37-39].

A significant feature of the multi-particle production process is the inherent fluctuation. Correlation analysis may yield valuable information about the dynamics of this process. In the past, for quite a few years, fluctuation and correlation had been analyzed extensively using novel perspective of studying non-statistical fluctuation which had resulted in rigorous interpretation of the pionization process. To begin with, Bialas and Peschanski [40] introduced a novel concept to analyze multiplicity fluctuations with respect to scaled factorial moments to identify and analyze the pattern of large non-statistical fluctuation, and finally heading towards the physical explanation of the origin of the fluctuations. They suggested that the character of the factorial moments is similar to the process of intermittency observed in hydrodynamics of turbulent fluid flow. Intermittency is a process which manifests prominent local fluctuations in consistent and large statistical systems. Moreover, it was noticed that multi-pion production process manifests a power-law trend of the factorial moments with respect to the magnitude of phase-space intervals in decreasing mode [40]. Bialas and Peschanski [40] also suggested a connection between intermittency and fractal behavior. Initially fractal pattern of multi-pion production process was analyzed from the context of intermittent fluctuations by utilizing the technique of factorial moment. Then a simple relationship was found between the intermittency indices and anomalous fractal dimension [41,42]. The inherent cascading process in the multi-pion production process naturally generates a fractal structure. In addition, the scale invariance existing in the process of hadronization was obvious from the spectrum of fractal dimensions. After that, innumerable methods based on the fractals had been applied to study the multi-pion production process using the parameters of $G$ and $T$ moments [43-47]. Then, different approaches like the Detrended Fluctuation Analysis (DFA), multifractal-DFA (MF-DFA) method [48,49] were implemented exhaustively for studying non-stationary, nonlinear data series to detect their long-range correlations. In the recent years, various studies based on the multi-fractal properties of the process of particle production had been reported [50-53]. In a number of recent works, self-similarity had been probed in the field of particle physics, like-in the Jet and Top-quark generation process at the experiments of Tevatron and LHC [54], in the process of strangeness production in $p p$ collisions at the experiments of Relativistic Heavy Ion Collider (RHIC) [55], in the proton spin phenomena and asymmetry of jet production process [56] to explain the collective phenomena [57] and in the implementation of the concept of self-similar symmetry of dark energy [58]. In a latest work [59], the fluctuation pattern inherent in the dynamics of particle production process in high energy collision has been analyzed using the MF-DFA method and also MF-DXA analysis using the pseudorapidity values of di-muon data extracted from the $p p$ collision at $7 \mathrm{TeV}$ and $8 \mathrm{TeV}$, respectively by CMS Collaboration [60,61].

A fundamental change in approach occurred with the latest advancements in the area of research using complex-network-based methodologies. Albert and Barabási have done a review in this area of complex-network analysis. There, they have also discussed the various scientific models and tools for random graphs, small-world and scale-free networks [62,63]. Havlin et al. have elaborated the application of network sciences to the analysis, comprehension, design and also restoration of multi-level complex systems [64]. Zhao et al. have applied correlation-based network method to 
analyze the dynamics of stock market and have been able to recognize global expansion and local clustering in the behavior of the market during crises, by applying the heterogeneous time scales [65]. In this context, Visibility Graph analysis method has attained its significance due its completely different and rigorous approach. Lacasa et al. have utilized fractional Brownian motion (fBm) and fractional Gaussian noises ( $\mathrm{fGn}$ ) series as a theoretical framework to examine real-time series in various scientific fields $[66,67]$. They have applied classical methodology of complex network to measure or quantify the long-range dependence and fractality of a time series. Using this method they converted $\mathrm{fBm}$ and $\mathrm{fGn}$ series into scale-free Visibility Graphs having degree distribution which are functions of the Hurst exponent. They have also studied the Stern series [68], the Q-series [69] and the Thue-Morse series [70], and it was revealed that the Visibility Graph method can distinguish different kinds of complexity, scale-freeness and fractal nature. They have also proposed that this algorithm is not only able to distinguish between random and chaotic series but also able to detect the spatial location of inverse bifurcations in chaotic dynamical systems. In the work [67], Lacasa et al. have established the robustness and reliability of Visibility Graph method with extensive simulations of artificial fractal data series and also of real and small data series having fractal dynamics reported in [71,72]. This method has also been implemented widely over time series with a finite number of data points and even with 400 data points [73], and has accomplished reliable result in different areas of science. Studies had been conducted in the area of the multiplicity fluctuation process in nucleus-nucleus and hadron-nucleus interactions, applying this Visibility Graph methodology in a few recent works [74-83]. Pb-Pb VSD masterclass data at $2.76 \mathrm{TeV}$ per nucleon pair from ALICE Collaboration [84] was used for scaling analysis of the pseudorapidity space, using both the method of complex-network-based Visibility Graph and MF-DFA [48,49], to study the prospective phase transition and the signature of quark-gluon plasma (QGP) in the work [82].

A recent review work has reported about the complexities involved in resonance production for various high energy collisions like $p p, p A$ and $A A$ collisions at LHC (using data from ALICE Collaboration), to understand the complexity and origin of different resonance states and eventually understand the inherent dynamics of the particle production process and the properties of the produced particles for the various collision systems [85]. In the work [21], CMS has presented a search for SUSY by applying an artificial natural network model to differentiate prospective SUSY signals from the background of SM. They implemented the search for SUSY in the events having pairs of oppositely signed leptons in the final state, hadronic jets and missing transverse energy, from the data of Run A (2011) of the $p p$ collision at $7 \mathrm{TeV}$ at the CMS detector. They have confirmed accordance between the expectations and observations of the SM which gives rise to more optimized supersymmetric standard models. Such successful attempts in this area of analysis of the pionization process in high energy interaction using chaos-based fractal, multi-fractal and complex-network procedure including attempts to probe the origin of the resonance states [85] as well as the use of new computational techniques such as neural networks in the area of high energy collisions have encouraged and inspired us to extend the methods to analyze another significant area of high energy interaction using a novel complex-network-based analysis method.

In this work we have tried to analyze the inherent symmetry scaling in the multi-particle production process using a novel method of the extended version of Visibility Graph algorithm which is Visibility Graph analysis in 3-dimentional space or 3D Visibility-Graph Analysis. The dataset (in totality) utilized for the search of SUSY using razor filter [34,35], has been generated from the multiJet primary $p p$ collision data of Run B of 2010 at $7 \mathrm{TeV}$ of the CMS Collaboration [36] is used for this analysis. The symmetry scaling for multi-particle production process in the case of SUSY is analyzed in terms of 3D-PSVG. The scaling analysis is based on the components of the $p_{T}$ in the $X, Y$ and $Z$ coordinates, extracted from the 4-momenta of the pair of mega-jets, obtained from the data of CMS Collaboration [36]. The signature of SUSY may be identified from the remarkable scaling behavior of the multi-particle production process observed in data of CMS Collaboration, indicated by 3D-PSVG, without using conventional invariant mass or $p_{T}$ techniques. 
The rest of the paper is structured as follows. Section 2 describes the methods of analysis in detail and the importance of various properties of scale-freeness. In Section 3, Section 3.1 elaborates the details of the data and Section 3.2 describes the detailed steps of our analysis and the relevant inferences. The test results are further analyzed with regards to the the physical relevance of the proposed parameters vis-a-vis the conventional ones used for analyzing SUSY in the high energy interactions in Section 4.

\section{Method of Analysis}

The complex-network-based methodology of Visibility Graph used to analyze the long-range correlation, fractality and the inherent scale-freeness of data series has been elaborated by Lacasa et al. [66,67].

As per this method, a data series $X=x_{1}, x_{2}, \ldots x_{i}, \ldots x_{n}$ is converted to its Visibility Graph, where let us suppose that $x_{i}$ is the $i^{\text {th }}$ data point. As shown in the Figure 1 , along the $X$-axis the sequence of nodes $i=1,2,3, \ldots$ are plotted, and magnitudes of $i^{\text {th }}$ node are plotted along the $Y$-axis. In this way we get a series of nodes corresponding to the data series. The data series $\left\{x_{i}\right\}$ for $i=1,2,3, \ldots$ is raised to the positive $Y$-axis to check the visibility between any pair of nodes.

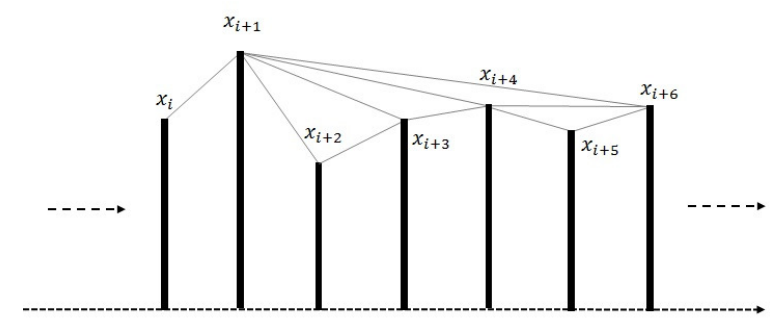

Figure 1. Visibility Graph for data series $x_{i}$ for $i=1,2,3, \ldots$

Any pairs of nodes are visible to each other via bi-directional edge if and only if the Equation (1) is satisfied. According to that we can see in the Figure 1, that the vertices or nodes $x_{p}$ and $x_{q}$, with $p=i$ and $q=i+6$, are visible to each other if and only if the Equation (1) is satisfied for all relevant $j$-s or all the nodes in sequence between $p=i$ and $q=i+6$. This is how the visibility is checked between any pair of nodes in the entire series and accordingly bi-directional edges are drawn and Visibility Graph of a data series is constructed. It is obvious that two consecutive nodes of the series are always visible to each other and hence consecutive nodes are always connected via a bi-directional edge.

$$
x_{p+j}<x_{q}+\left(\frac{q-(p+j)}{q-p}\right) \cdot\left(x_{p}-x_{q}\right)
$$

where $\forall j \in Z^{+}$and $j<(q-p)$.

In this work we have proposed a novel method of 3D Visibility Graph based on the method of identifying bi-directional edges of visibility between a pair of nodes plotted according to the data-values of the points of one-dimensional self-similar data series, prescribed by Lacasa et al. [66,67]. In the proposed method, the scaling analysis is done in 3-dimentional space constructed out of the components of the $p_{T}$ in the $X, Y$ and $Z$ coordinates, extracted from 4-momenta of the signatures of final state of the pair of mega-jet, obtained from the data of CMS Collaboration [36].

The proposed method of constructing the 3D Visibility Graph is described in detail in Section 2.1 and the method of deriving the scaling exponent is elaborated in Section 2.2.

\subsection{D Visibility Graph Algorithm}

In this method we have a a set of $\{(x, y, z)\}$ values. We have plotted them in $(X, Y, Z)$ space of real numbers. For every $(x, y, z)$ value, $x, y, z \in \mathbb{R}$. However, the $z$-values are raised to the positive $Z$-axis 
above the $(X, Y)$-plane while plotting the node during the visibility-check of a pair of nodes/vertices and not the bases or the $(x, y)$-values in the $(X, Y)$-plane, in $(X, Y, Z)$ space. Hence, for each $(x, y, z)$ value for each set of $x, y \in \mathbb{R}$ plotted in $(X, Y)$-plane the corresponding $z$ is $\in \mathbb{R}^{+}$. Now for each pair of nodes in the $(X, Y, Z)$ space, first the coordinates in the $(X, Y)$-plane corresponding to the pair of $(x, y, z)$-values are checked, whether they satisfy Equation (2) or not.

For instance, while checking the visibility between the nodes $\left(x_{p}, y_{p}, z_{p}\right)$ and $\left(x_{q}, y_{q}, z_{q}\right)$, the first step is to check if all the points between them in terms of $X$ and $Y$ coordinates satisfy the Equation (2) or not. In Equation (2), $j$ is the index of all the points between $\left(x_{p}, y_{p}\right)$ and $\left(x_{q}, y_{q}\right)$. If none of the $j^{t h}$ points for $j \in(q-p)$ and $j<(q-p)$ satisfy Equation (2), then nodes $\left(x_{p}, y_{p}, z_{p}\right)$ and $\left(x_{q}, y_{q}, z_{q}\right)$ are visible to each other and they will be connected via a bi-directional edge. In these cases, Equation (3) will not be checked. If there exists at least 1 point (or index $j \in(q-p)$ and $j<(q-p)$ ) between $\left(x_{p}, y_{p}\right)$ and $\left(x_{q}, y_{q}\right)$ (corresponding to $\left(x_{p}, y_{p}, z_{p}\right)$ and $\left.\left(x_{q}, y_{q}, z_{q}\right)\right)$ for which Equation (2) is satisfied then only we move on to check the next Equation (3) for existence of visibility between the nodes $\left(x_{p}, y_{p}, z_{p}\right)$ and $\left(x_{q}, y_{q}, z_{q}\right)$.

$$
\frac{x_{p+j}-x_{p}}{x_{q}-x_{p}}=\frac{y_{p+j}-y_{p}}{y_{q}-y_{p}}
$$

where $\forall j \in Z^{+}$and $j<(q-p)$.

All the points $(\{(x, y, z)\})$ satisfying the Equation (2), in between the pair of $(x, y, z)$-values in question, are noted. Then only for all those values, the $z$ coordinates are noted and for each of the corresponding $z$-values, Equation (3) is checked. If for each of these $z$ coordinates, the Equation (3) is satisfied, then only the pair of $(x, y, z)$-values in question are visible to each other and they will be connected via a bi-directional edge.

For instance, to check visibility between the nodes $\left(x_{p}, y_{p}, z_{p}\right)$ and $\left(x_{q}, y_{q}, z_{q}\right)$ for all the points with index $k \in j$ for which $\left(x_{k}, y_{k}\right)$ satisfies the Equation (2), the corresponding $z$ coordinates are checked with the Equation (3). If and only if all the corresponding $z_{k}$ s satisfy Equation (3), then only there will be existence of visibility between the nodes $\left(x_{p}, y_{p}, z_{p}\right)$ and $\left(x_{q}, y_{q}, z_{q}\right)$.

$$
z_{p+k}<z_{q}+\left(\frac{q-(p+k)}{q-p}\right) \cdot\left(z_{p}-z_{q}\right)
$$

where $\forall k \in Z^{+}$and $k \in$ [all values of the index for which $\left(x_{k}, y_{k}\right)$ satisfies the Equation (2).

For the visibility graphs constructed out of one-dimensional self-similar data series, as per the method of Lacasa et al. [66,67], two consecutive nodes of the series are always visible to each other and hence consecutive nodes are always connected via a bi-directional edge. Similarly, in the 3D Visibility Graph constructed out of $\{(x, y, z)\}$-values in $(X, Y, Z)$ space, for each vertices the adjacent nodes in all the directions in $(X, Y)$ space are visible from it and hence from all those nodes/vertices there are bi-directional edges with the node in question.

Figure 2, shows a part of 3D Visibility Graph showing 5 nodes with indexes $i \in[i, i+1, \ldots i+4]$ for the purpose of illustration. According to this 3D Visibility-Graph Algorithm, in Figure 2 the vertices or nodes $\left(x_{p}, y_{p}, z_{p}\right)$ and $\left(x_{q}, y_{q}, z_{q}\right)$, with $p=i$ and $q=i+3$, have 2 vertices in between with indices $i+1, i+2$. The node $\left(x_{i+1}, y_{i+1}, z_{i+1}\right)$ satisfies Equation (2) for its $\left(x_{i+1}, y_{i+1}\right)$ but the node $\left(x_{i+2}, y_{i+2}, z_{i+2}\right)$ does not satisfy Equation (2). Hence, the $z$ coordinate is checked with the Equation (3), for the node $\left(x_{i+1}, y_{i+1}, z_{i+1}\right)$ and not for the next node $\left(x_{i+2}, y_{i+2}, z_{i+2}\right)$ but this node does not also obstruct visibility between the pair of nodes in question. The node $\left(x_{i+1}, y_{i+1}, z_{i+1}\right)$ does not satisfy the Equation (3) with respect to its $z$ coordinate and hence obstruct visibility between the nodes $\left(x_{p}, y_{p}, z_{p}\right)$ and $\left(x_{q}, y_{q}, z_{q}\right)$. Finally, we can conclude that nodes $\left(x_{p}, y_{p}, z_{p}\right)$ and $\left(x_{q}, y_{q}, z_{q}\right)$, with $p=i$ and $q=i+3$ are not visible to each other. 


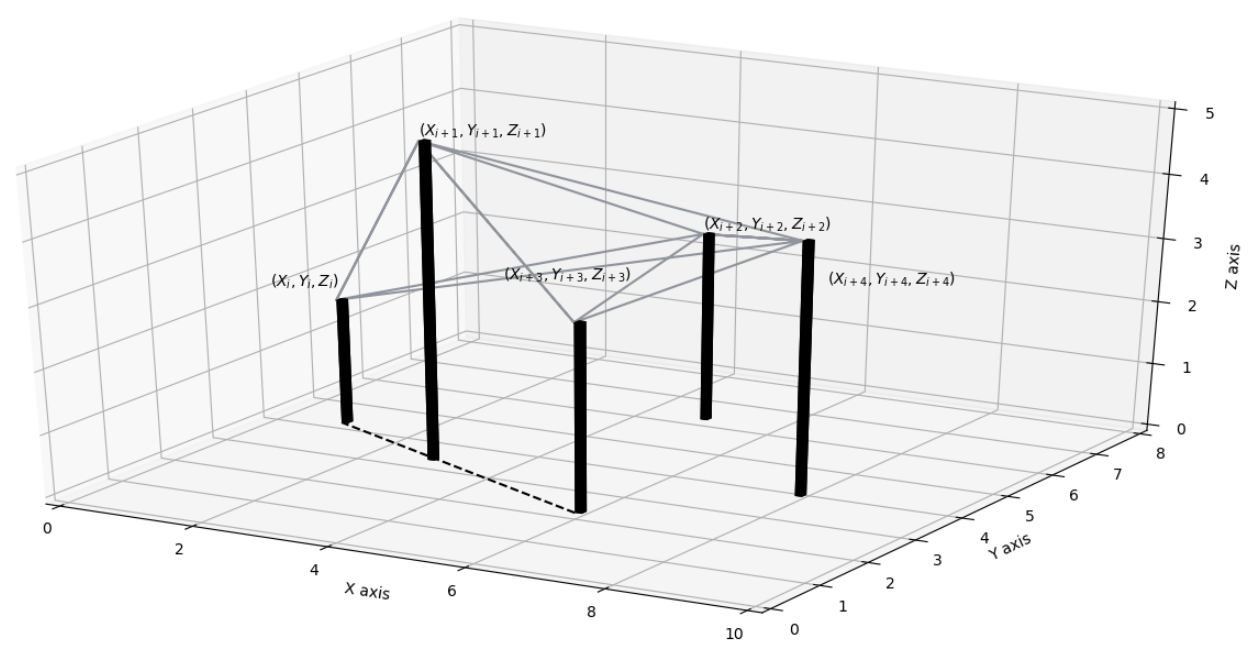

Figure 2. A part of the 3D Visibility Graph constructed out of a set of $\{(x, y, z)\}$-values.

For another pair of nodes, $\left(x_{p}, y_{p}, z_{p}\right)$ and $\left(x_{q}, y_{q}, z_{q}\right)$, with $p=i+1$ and $q=i+4$, none of the nodes in between with index $i+2, i+3$, satisfy Equation (2) with respect to their $X$ and $Y$ coordinates. Hence, for none of these intermediate nodes, the Equation (3) is checked and we can conclude that $\left(x_{p}, y_{p}, z_{p}\right)$ and $\left(x_{q}, y_{q}, z_{q}\right)$ are visible to each other and have a bi-directional edge between them.

Figure 2 also shows that each node has visibility and a bi-directional edge with each of its neighboring nodes.

Following this method, the visibility between any pair of nodes in the entire 3-dimentional space is checked and bi-directional edges are made according to the visibility and a 3D Visibility Graph is constructed from a data space. In this way, a data space is converted to its corresponding Visibility Graph.

For both One and 3D Visibility Graphs, it should be emphasized that as per these methods, the visibility between two nodes in the Visibility Graph constructed out of data series (for one dimensional series) or data spaces (for 3-dimentional spaces) does not change significantly if the number of nodes between the corresponding nodes is increased beyond a certain limit. In effect, both One and 3D Visibility Graph analysis generates nearly uniform results if the dimensions of the data series or data spaces of analyses are raised above that particular limit. Jiang et al. have analyzed data series of various length and inferred that length of data series above specific number limit does not have a substantial effect on the degree distribution of the graph. They have applied this methodology on data series of the human heartbeat having a finite number of data points and even with 400 data points steady and consistent results [73] were achieved.

\subsection{D Power-of-Scale-Freeness-of-Visibility-Graph (3D-PSVG)}

The degree of a node or vertex in a graph (here Visibility Graph) is defined as the number of edges the node has with respect to the rest of the nodes in the graph. The visibility between a pair of nodes and the the presence of bi-directional edge between them is deciphered as per the process described in the Section 2.1 for a 3-dimentional space.

The degree distribution, here denoted by $P(k)$, of the graph is defined as the fraction of nodes having the degree $k$, with respect to the total number of nodes in the graph (for both One and 3D Visibility Graphs). Therefore, if $n_{k}$ denotes the number of nodes in the graph having the degree of $k$ and if the total number of nodes in the graph is denoted by $n$, then $P(k)$ is defined by $P(k)=n_{k} / n$ for all probable values of $k$.

As fractal data series is converted into a scale-free Visibility Graph [66], $P(k) \sim k^{-\lambda_{p}}$ for a Visibility Graph, where $k$ is every possible degree of the nodes of the graph and $\lambda_{p}$ is constant. If the data series 
or data space has inherent long-range correlation, fractality and scale-freeness, the degree distribution of the vertices/nodes of the Visibility Graph is constructed out of the data series or space which obeys the so-called power-law characterizing the inherent scale-freeness. Then the scaling exponent denoted by $\lambda_{p}$, is termed as the Power of the Scale-freeness in Visibility Graph (PSVG) for one dimensional data series and $3 D-P S V G$ for 3-dimentional data spaces. This scaling exponent or degree of scale-freeness of a Visibility Graph denotes the degree of complexity and fractal characteristic of data $[66,67,86]$. As the PSVG or 3D-PSVG is the estimation of complexity, self-similarity, either of them may denote the Fractal Dimension (FD) of the data. Further in the work [67], Lacasa et al. have also inferred that the Hurst exponent and $P S V G$ are related in an inverse-linear manner.

\section{Experimental Details}

The experimental primary dataset used for the proposed analysis is a publicly available dataset from the CMS Collaboration. The details of data and its extraction process are elaborated in Section 3.1 and the detailed method of the experiment is elaborated in a step-by-step manner in Section 3.2.

\subsection{Data Description}

The multiJet primary dataset of $p p$ collisions in AOD format of Run B of 2010 at $7 \mathrm{TeV}$ [36] of the CMS Collaboration is extracted and considered as the source dataset of the first level of this experiment. Stored events in this primary dataset were qualified to be selected due of the presence of two or more jets with high energy, and having a high HT, which is the sum of the energy of all the jets produced in the event.

During the data selection procedure all the runs recorded by the CMS Collaboration are qualified as good for physics analysis if all sub-detectors, trigger (an algorithm to select particle collisions to be stored in the primary dataset and rest are discarded), lumi and physics particles like electron, muon, photon, jet, etc. show the expected performance. First level of validation is done based on the evaluations of offline shifters and then the feedback is taken from the detector and experts from Physics Object Group (POG). All of this information is stored in a specific database named Run Registry. The CMS Data Quality Monitoring group validates the integrity of the certification and creates a (.json) file of qualified runs to be utilized for physics analysis. The (.json) file [87] luminosity sections containing the good runs which have been selected from the primary dataset of events for further processing. This is the second level of selection. Then the filter is run over the multiJet primary dataset from the CMS open data, after the second level of selection, and the events with jet candidates with certain parameters having the required threshold values are selected and resultant files in (.root) and (.csv) formats are created. The jets are reconstructed and grouped into two mega-jets-one leading mega-jet with the largest $p_{T}$ and another subleading mega-jet with the largest $p_{T}$. The mega-jets are derived as sum of the 4-momenta of their constituent particles. From the reconstructed jets of the events with a large degree of missing transverse energy, the razor variables $M_{R}$ and $R^{2}$ are calculated for use in LSP searches [35]. This is the final level of selection. In this analysis, we have used this set of $X, Y$ and $Z$ components of the $p_{T}$ extracted from the 4-momenta of the pair of mega-jets.

The required programs and scripts are publicly available in CernVirtual Machine [88] which uses Scientific Linux CERN 5 (slc5). For data extraction in this manuscript, we have used CMS open data from CMS Virtual Machine (VM). This VM image provides the CMS computing environment to be used with the 2010 CMS open data [89]. We have used the Razor filter and analyzer for SUSY search programs and scripts provided in this CMS VM environment in the software CMSSW_4_2_8 as per our requirements and created the resultant files in (.root) and (.csv) formats. Then the components in $X, Y$ and $Z$ coordinates of the $p_{T}$ taken out from the 4-momenta of the pair of mega-jets, are extracted from the generated resultant datasets. The details of the programs [35] and software [90] are publicly available.

We have extracted around 30,000 events in the output dataset generated from the multiJet primary dataset of the CMS Collaboration, after three levels of filtering as per the process stated above. 
It must be noted that we have applied the proposed approach of 3D Visibility Graph method using the components in $X, Y$ and $Z$ coordinates of the $p_{T}$ of the reconstructed mega-jets for each of the extracted events. There are a couple of mega-jets corresponding to each event. Hence, from each extracted event-record, a couple of $p_{T}$ values corresponding to a couple of mega-jets, is obtained. Hence, the length of fundamental experimental datasets (containing $X, Y$ and $Z$ coordinate values of $p_{T}$ of each of the couple of mega-jet) is around $2 * 30,000 \approx 60,000$ for each of the $X, Y$ and $Z$ coordinate values. We have already elaborated in the Section 2.1 that the Visibility Graph method gives reliable results for datasets with substantially small length, and even with a dataset of length 400 [73]. Moreover, we have also applied this method in the area of high energy interaction with a finite number of events e.g., [78,79,81-83], etc. and have been able to obtain reliable results. Hence, it can be concluded that the size of experimental data space is large enough to give reliable results.

\subsection{Data Analysis and Results}

1. The set of components of the $p_{T}$ in $X, Y$ and $Z$ coordinates, is created from the 4-momenta of the pair of mega-jets of the resultant dataset created out of the multijet primary dataset of Run $\mathrm{B}$ of 2010 at $7 \mathrm{TeV}$ [36] for supersymmetric data of the CMS Collaboration, as per the method described in Section 3.1. This way we get a set of $(x, y, z)$-values in 3-dimentional $(X, Y, Z)$ space. Then we create 3 sets of $3 \mathrm{D}(X, Y, Z)$ spaces defined following the instructions described below.

(a) The components of the $p_{T}$ in $Y$ coordinate are taken as the set of $x$-values, corresponding $Z$ coordinate values are taken as set of $y$-values and $X$ coordinate values are taken as set of $z$-values. This way we get a 3-dimentional space made up of the set of $(x, y, z)$-values which actually corresponds to $Y, Z$ and $X$ coordinate components of the $p_{T}$ values. We denote this data space as $[y, z, x]_{s s}$. Figure 3 shows the pattern of the data space $[y, z, x]_{s s}$.

(b) The components of the $p_{T}$ in the $Z$ coordinate are taken as the set of $x$-values, corresponding $X$ coordinate values are taken as set of $y$-values and $Y$ coordinate values are taken as set of $z$-values. This way we get a 3-dimentional space made up of the set of $(z, x, y)$-values which actually corresponds to $Z, X$ and $Y$ coordinate components of the $p_{T}$ values. We denote this data space as $[z, x, y]_{s s}$. Figure 4 shows the pattern of the data space $[z, x, y]_{s s}$.

(c) The components of the $p_{T}$ in the $X$ coordinate are taken as the set of $x$-values, corresponding $Y$ coordinate values are taken as set of $y$-values and $Z$ coordinate values are taken as set of $z$-values. This way we get a 3-dimentional space made up of the set of $(x, y, z)$-values which actually corresponds to $X, Y$ and $Z$ coordinate components of the $p_{T}$ values. We denote this data space as $[x, y, z]_{s s}$. Figure 5 shows the pattern of the data space $[x, y, z]_{s s}$

Then, for each of the 3 data spaces, a 3D Visibility Graph is constructed as per the method described in Section 2.1. After that, $P(k)$ versus $k$ datasets are created from the degree distribution of each of the graphs for all probable values of $k$ as per the method described in Section 2.2. The observations from the scaling analysis are as follows: 


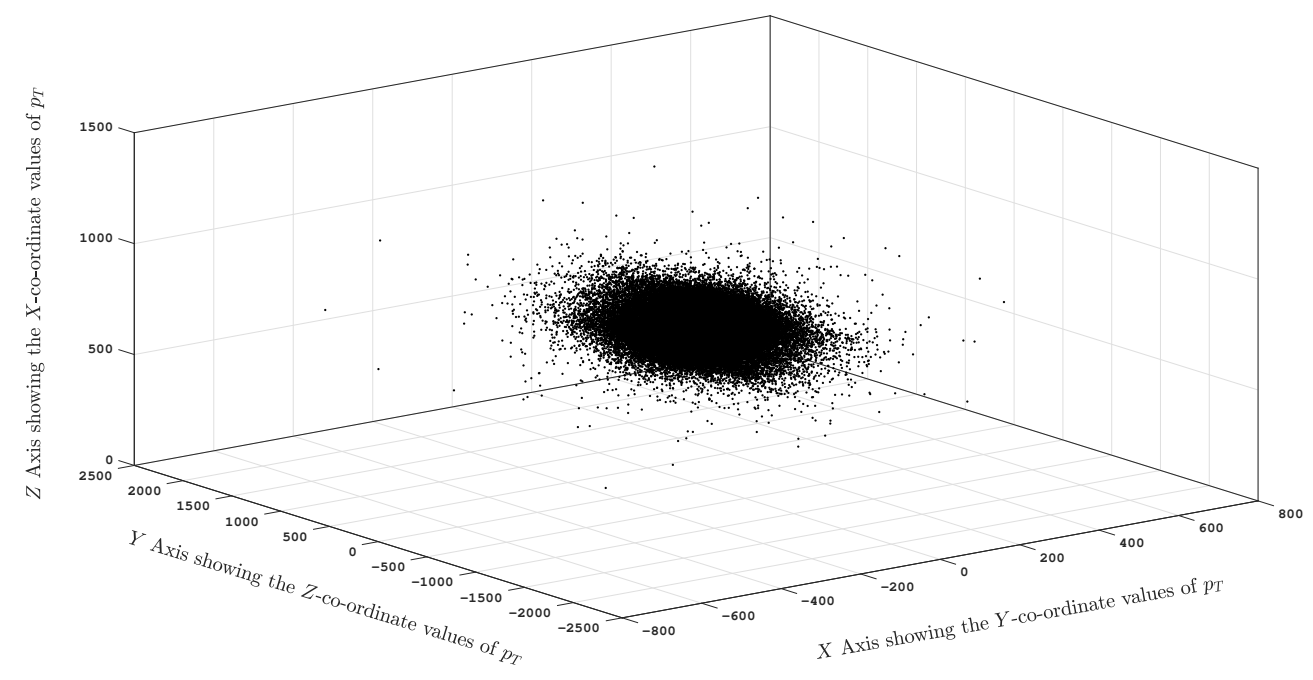

Figure 3. Data space- $[y, z, x]_{s s}$.

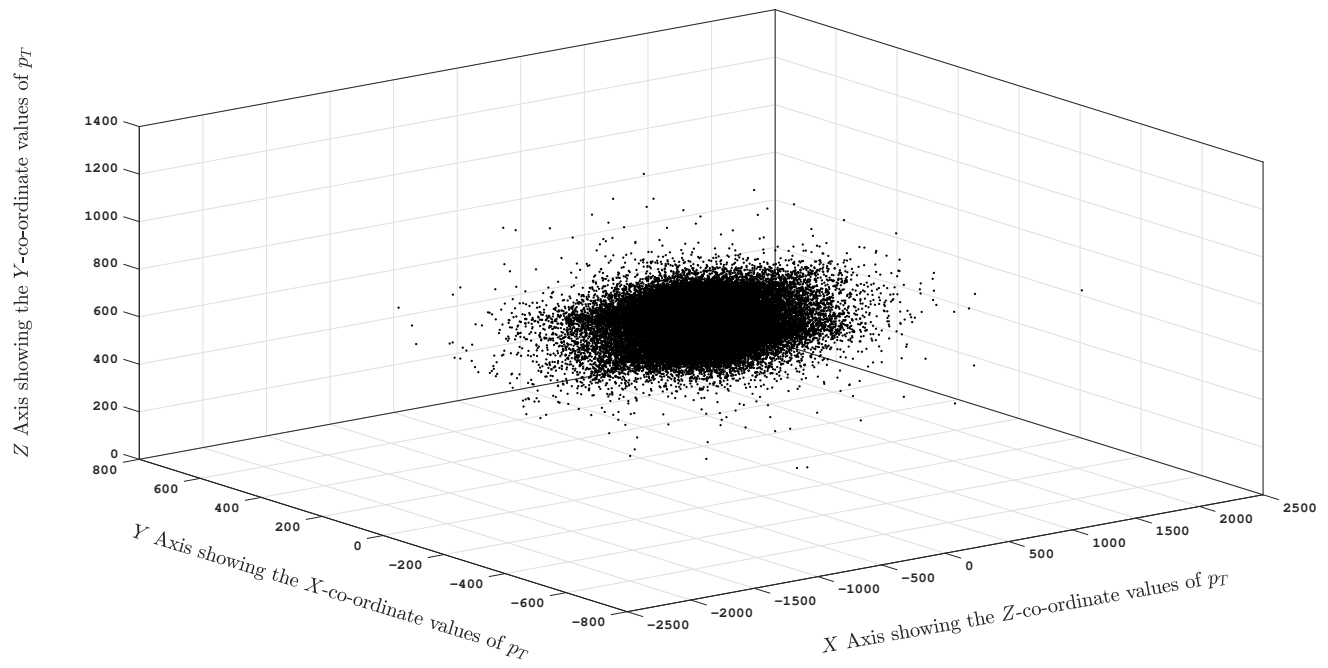

Figure 4. Data space- $[z, x, y]_{s s}$.

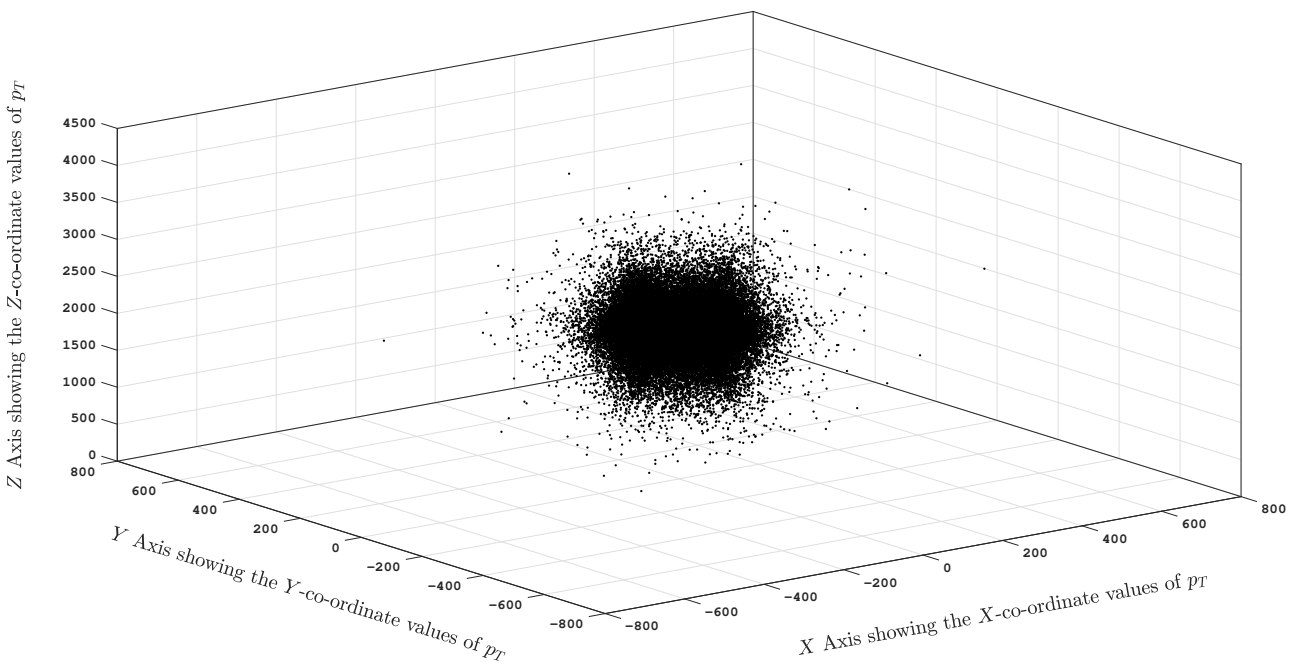

Figure 5. Data space- $[x, y, z]_{s s}$. 
2. Figure 6a shows the trend of $P(k)$ with respect to corresponding $k$ s and Figure $6 \mathrm{~b}$ shows the trend of $P(k)$ with respect to corresponding $1 / k$ s in log-log scale generated from the 3D Visibility Graph for the data space $[y, z, x]_{s s}$. Similarly, Figure $7 \mathrm{a}, \mathrm{b}$ for the trend of $P(k)$ versus $k$ and $P(k)$ versus $1 / k$ in $\log$-log scale for the 3D Visibility Graph for the data space $[z, x, y]_{s s}$. Figure $8 \mathrm{a}, \mathrm{b}$ for the trend of $P(k)$ versus $k$ and $P(k)$ versus $1 / k$ in log-log scale for the 3D Visibility Graph for the data space $[x, y, z]_{s s}$. Table 1 shows the comparison of 3D-PSVG values and other relevant details calculated for the 3 data spaces.

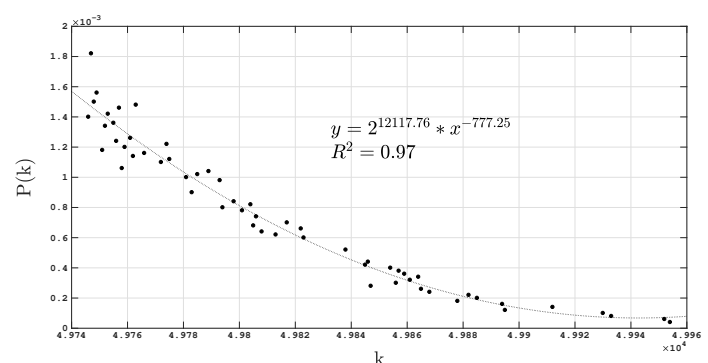

(a)

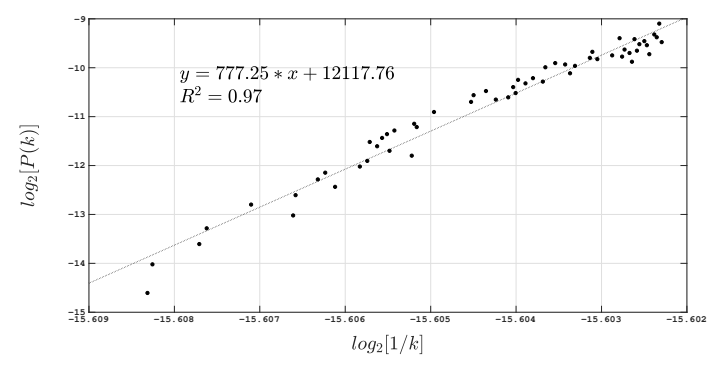

(b)

Figure 6. (a) $P(k)$ vs. $k$ plot and (b) $\log _{2}[P(k)]$ vs. $\log _{2}(1 / k)$ plot generated from the $3 \mathrm{D}$ Visibility Graph for the data space $[y, z, x]_{s s} . R^{2}$ is a statistical measure of fit that indicates how much variation of a dependent variable is explained by the independent variable(s) in a regression model.

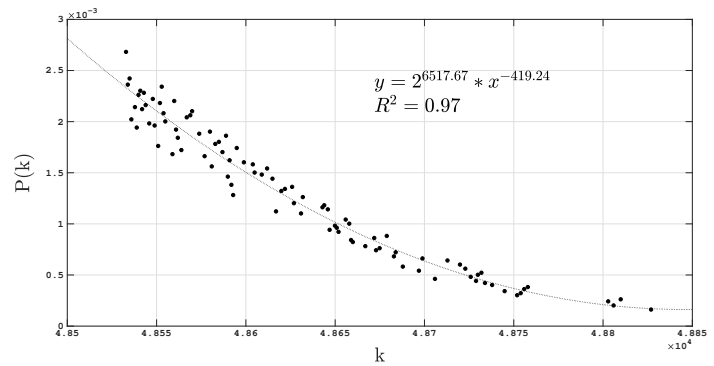

(a)

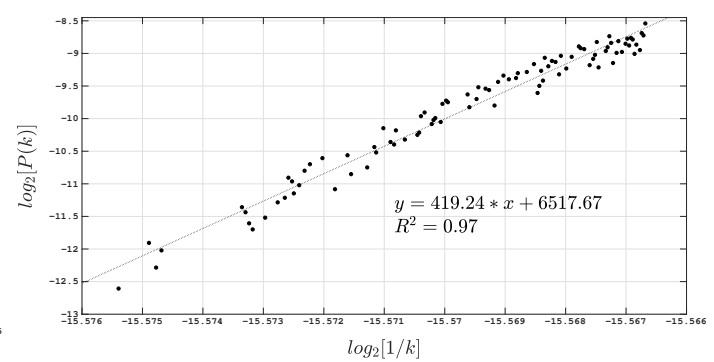

(b)

Figure 7. (a) $P(k)$ vs. $k$ plot and (b) $\log _{2}[P(k)]$ vs. $\log _{2}(1 / k)$ plot generated from the $3 D$ Visibility Graph for the data space $[z, x, y]_{s s} . R^{2}$ is a statistical measure of fit that indicates how much variation of a dependent variable is explained by the independent variable(s) in a regression model.

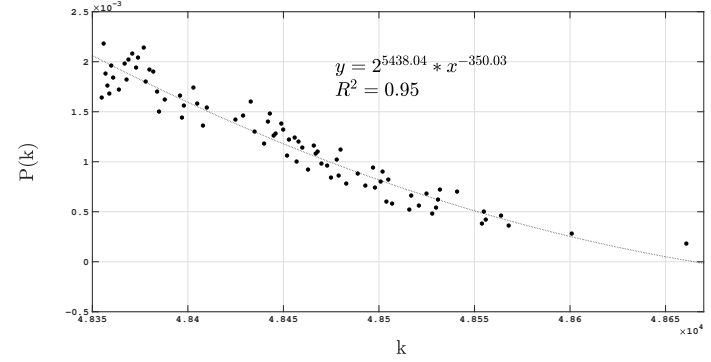

(a)

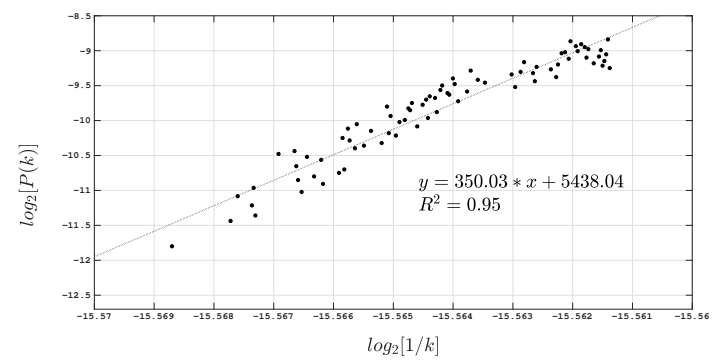

(b)

Figure 8. (a) $P(k)$ vs. $k$ plot and (b) $\log _{2}[P(k)]$ vs. $\log _{2}(1 / k)$ plot generated from the 3D Visibility Graph for the data space $[x, y, z]_{s s} . R^{2}$ is a statistical measure of fit that indicates how much variation of a dependent variable is explained by the independent variable(s) in a regression model.

3. The power-law fitting for the set of $P(k)$-values and the corresponding $k$-values is performed as per the method prescribed by Clauset et al. [91]. As it has been already elaborated, each of the 3 sets of $k$-values is the probable degree of the nodes of the 3D Visibility Graph generated from each of the 3 data spaces. In other words, each $P(k)$ versus $k$ series is the degree distribution of the corresponding 3D Visibility Graph. Here, the method of maximum-likelihood-estimation 
(MLE) is applied to extract the power-law exponent from the best-fitted curve according to the method suggested by Clauset et al. [91]. We have followed the method to estimate the lower bound on power-law behavior as per the method in [91] to calculate the value of $k_{\min }$ for which all the $k>=k_{\min }, P(k)$ versus $k$ series are analyzed for the trend of power-law. After that, the performance of the power-law model constructed with the deduced $k_{\min }$ as per the instructions in [91] is validated with the help of the Kolmogorov-Smirnov (KS) test with that specific $k_{\min }$ that minimizes the KS statistics for the corresponding $P(k)$ versus $k$ dataset. The maximum value of absolute distance denoted by $D_{\text {crit }}$ is estimated as the difference between the value of cumulative distribution function estimated for the actual observations with $k_{\min }$ as the minimum value and the value calculated with the best fitted power-law model with all the observations $>k_{\min }$, to calculate KS statistics. $k_{\min }$ is finalized as the one which minimizes $D_{\text {crit }}$ according to KS table as per the level of significance 0.10 [91]. In this process, the value of $k_{\min }$ is derived and then the power-law exponent of the best-fitted model distribution is calculated as per the method of MLE. We have finalized the calculated value of $k_{\min }$ and the corresponding power-law exponent by plotting them in log-linear scale as per the instructions in [91]. Following this method, first the value of $k_{\min }$ and then the power-law exponent are deduced for each of the 3 3D Visibility Graphs constructed out of the data spaces as elaborated in Step 1.

After that, the goodness-of-fit parameter is calculated for the best fit of the power-law distribution, deduced with the help of $k_{\min }$ and the power-law exponent, with respect to the observed data or the extracted $P(k)$ versus $k$ distribution of the 3D Visibility Graphs. The goodness-of-fit parameter is calculated as per the method of KS test for goodness-of-fit described in [91] with the following parameters deduced as per the method already elaborated.

- The power-law exponent calculated by MLE method.

- Derived $k_{\min }$ minimizing the KS statistics.

The power-law distribution created with the above two parameters proves to be the best-fitted model as the estimated $p$-value is found to be significantly greater than the significance level of 0.10 , taken as per [91], for all the 3 data spaces. In addition, for all the 3 data spaces the power-law relationship is also established by substantially high values of $R^{2}$. Here, the (KS test is repeated to verify for goodness-of-fit parameter with respect to observed data and for a generated set of 2000 synthetic datasets produced from a random power-law distribution with the same set of calculated parameters of power-law exponent and $k_{\min }$. Around $75-85 \%$ of the datasets have not successfully discarded the null hypothesis-the observed data conforms to a power-law distribution with the calculated parameters of power-law exponent and $k_{\min }$. The $p$-value derived from this testing is $\approx 0.75-0.85$, which is far more than 0.10 (the level of significance to be taken as per [91]). From this, it can be concluded that the observed $P(k)$ versus $k$ distribution is a power-law distribution with the deduced power-law exponent. The 3D-PSVG of each of the 3 3D Visibility Graphs is derived from the power-law exponent and they are listed in Table 1 along with their corresponding standard error, $\chi_{\exp }^{2} / \mathrm{DOF}$ and $R^{2}$-values.

Table 1. Comparison of 3D-PSVG values and other relevant details calculated for the 3 data spaces.

\begin{tabular}{cccc}
\hline Data Space & 3D-PSVG & $\chi_{\text {exp }}^{\mathbf{2}}$ /DOF & $\boldsymbol{R}^{\mathbf{2}}$ \\
\hline$[y, z, x]_{s S}$ & $777.25 \pm 0.25$ & 0.02 & 0.97 \\
{$[z, x, y]_{s s}$} & $419.24 \pm 0.17$ & 0.02 & 0.97 \\
{$[x, y, z]_{s S}$} & $350.03 \pm 0.22$ & 0.01 & 0.95 \\
\hline
\end{tabular}

The power-law behavior of the corresponding $P(k)$ versus $k$ distribution, can be confirmed from the high range of $p$-values and high values of $R^{2}$ which are shown in the Table 1 and the Figures $6 \mathrm{a}, 7 \mathrm{a}$ and $8 \mathrm{a}$ for all the 3 data spaces. 3D-PSVGs, are also extracted from the slope 
estimated from straight-line fitting performed for the corresponding $\log _{2}[P(k)]$ versus $\log _{2}(1 / k)$ for all the three 3D Visibility Graphs. They are shown along with the same values of $R^{2}$ (estimated for straight-line fitting) in Figures $6 \mathrm{~b}, 7 \mathrm{~b}$ and $8 \mathrm{~b}$, respectively. We have already elaborated in Section 2.2 that if a data space has inherent long-range correlation, fractality and scale-freeness, the degree distribution of the vertices/nodes of that 3D Visibility Graph generated from the data space obeys the power-law which in turn denotes the inherent scale-freeness. Hence, high range of $p$-values and high values of $R^{2}$ of the power-law fitting of the degree distribution of the 3D Visibility Graphs signify the scale-freeness inherent in the supersymmetric data represented in terms of components of $p_{T}$ of produced pairs of mega-jets selected from events in $p p$ collisions of Run B of 2010 at $7 \mathrm{TeV}$ [36] of the CMS Collaboration, generated from 3 perspectives- $(y, z, x)$, $(z, x, y)$ and $(x, y, z)$ as elaborated in Step 1 .

It must also be noted that 3D-PSVG values obtained from power-law exponents (derived from $P(k)$ versus $k$ distribution) or slopes of straight-line fitting (derived from $\log _{2}[P(k)]$ versus $\log _{2}(1 / k)$ trend) correspond to the degree of complexity and fractality of the data spaces.

4. It must be noted that as opposed to the One-Dimensional Visibility Graph, the 3D Visibility Graph construction method has two levels of validation for a couple of nodes to be visible to each other or to be connected via a bi-directional edge. In the case of a 3D Visibility Graph, for many of the pairs of nodes there are no nodes between them in the $X, Y$-plane which means none of the nodes between the pair of nodes in the $X, Y$-plane satisfy the Equation (2) to obstruct visibility. Hence, the values of $k$ or every possible number of edges the nodes have with rest of the nodes in the 3D Visibility Graph start from a higher value, whereas the degree distribution or the $P(k)$ s for the corresponding $k$ s are of substantially low value. This results in a substantially higher value of intercept as well as the power-law exponent.

However, for the couple of nodes for which all the nodes in between satisfy both the Equation (2) and then the Equation (3) to become visible to each other and get connected via bi-directional edge, the number of edges $(k)$ and their corresponding degree distribution $(P(k))$ give rise to a power-law distribution having high range of $p$-values [91] and high values of $R^{2}$ and hence a significantly higher degree of goodness-of-fit for the data spaces corresponding to the supersymmetric data. This implies the presence of strong inherent scale-freeness and self-similarity deeply embedded in the supersymmetric data which is revealed after two levels of checking via rigorous and complex-network-based method of the 3D Visibility Graph, that too by analyzing the components in $X, Y$ and $Z$ coordinates of a single variable of $p_{T}$ of produced pair of mega-jets.

\section{Conclusions}

SUSY has been been an area of enormous interest for the past 60 years as it is the most encouraging theory in the vast area of high energy physics. The LHC has been empowering ATLAS and CMS Collaborations in an attempt to prove SUSY in various high energy interactions of $p p$ collisions at $7,8,13 \mathrm{TeV}$ and so on. Thousands of searches have been performed in an attempt to empower our understanding of nature. For the last few decades, a significant amount of missing transverse energy has been analyzed as the most prospective observable for identifying the production and decay of LSP at various experiments of the colliders. The SM of SUSY which obeys the $R$-parity conservation, predicts the signatures with jets arising out of decaying pairs of squarks or gluinos (supersymmetric partners of quarks and gluons), and a substantial amount of missing transverse energy originating from undetected weakly communicating lightest LSP, at the LHC. However, searches for such events have not been quite encouraging in displaying the signature of SUSY and also have not yielded the strongest bounds as per the considered details of the supersymmetric models. The majority of these limits or bounds thus derived are considered as lower limit for the mass parameter. It has been inferred that supersymmetric partners must possess masses higher than the limits obtained by the 
analysis. However, it was also concluded that these limits are not totally definitive and rather they are dependent on the other features or properties of the SUSY. To summarize, it can be inferred that most of these decay processes have a common and distinct feature among themselves-the existence of numerous jets of high energy arising from the anticipated high degree of difference between gluinos and the so-called daughter particles, and evidently, a substantial amount of missing transverse energy originating from the couple of high momentum LSPs which get away with detection. There have been continuous searches for SUSY at the LHC, for both prompt, non-prompt, for R-parity conserving as well as $R$-parity violating production and decays. It is concluded from the results of these searches that LHC has excluded the existence of gluinos below $2 \mathrm{TeV}$, and existence of stops and gauginos below $1 \mathrm{TeV}$. These limits or checks conveyed greater possibilities of further experiments at the collider. However, these signatures of LSP are usually obtained under the assumption of bit optimistic situations where sparticles' decay into the final states is studied with a 100\% branching fraction. The fact is that SUSY might have been in a camouflaged state at lower mass-scales as a consequence of difficult and rather challenging mass-spectra and also mixed modes of decays.

In this analysis, we have proposed a novel, chaos-based and complex-network-based method of 3D Visibility Graphs, where the visibility of two nodes in a 3-dimentional space is derived after two-step verification. This way, the inherent scale-freeness and self-similarity, if it at all exist, can be identified to the deepest level in a rigorous way. It has already been elaborated in Section 3.1 that the multiJet primary dataset ( $p p$ collisions dataset from Run B of 2010 at $7 \mathrm{TeV}$ [36]) of the CMS open data is filtered. It is based on certain criteria to calculate the razor variables $M_{R}$ and $R^{2}$ which have been used in LSP searches [35]. Shape analysis has been performed in the razor plane made up of $M_{R}$ and $R^{2}$ and experimental data have been validated against a background model made up of simulated events [36]. In the proposed analysis, a 3-dimentional data space is made up of components in $X, Y$ and $Z$ coordinates of the $p_{T}$ extracted from 4-momenta of the pair of mega-jets of the events selected from the same multiJet primary dataset and 3D Visibility Graph analysis has been done over. In the proposed analysis, all the data points have been taken from the multiJet dataset of CMS and no simulated dataset has been used. The summary of inferences are listed below.

1. The number of edges $(k)$ and their corresponding degree distribution $(P(k))$ for the 3D Visibility Graphs constructed from supersymmetric data space from 3 perspectives- $(y, z, x),(z, x, y)$ and $(x, y, z)$ as elaborated in Step 1 in Section 3.2, are analyzed for fitting with regard to power-law distribution. The trend of $P(k)$ versus $k$ can be examined from Table 1 and the Figures 6-8a for all the 3 data spaces. A high range of $p$-values [91] and high values of $R^{2}$ and hence high degree of goodness-of-fit are obtained for the power-law fitting for $k$ and their corresponding degree distribution $P(k)$ for all the 3 data spaces signify the presence of high degree of inherent scale-freeness and self-similarity in the supersymmetric data spaces. This scale-freeness and self-similarity of the data space is captured from their manifestation through the $p_{T}$-space embedded in 4-momenta of the pair of mega-jets produced from the $p p$ collisions of Run B of 2010 at $7 \mathrm{TeV}$ [36] of the CMS Collaboration.

2. As per the proposed 3D Visibility Graph method, visibility between any two nodes is decided after two steps of validation through Equations (2) and (3). A substantial number of pairs of nodes become visible to each other since the nodes in between do not satisfy Equation (2) and hence do not obstruct visibility as per the method explained in Section 2.1. This leads to significantly higher values of intercept and the power-law exponent 3D-PSVG for the experimental data spaces. It is to be noted that even after two levels of validation the proposed method has been successful in extracting the inherent degree of complexity and self-similarity out of supersymmetric data from a single variable of $p_{T}$ of the produced pair of mega-jets, in the most rigorous manner.

3. The power-law exponent 3D-PSVG for the supersymmetric data space from 3 perspectives, is derived by power-law fitting done for the $P(k)$ versus $k$ distribution and observed through the slopes of straight-line fitting done for corresponding $\log _{2}[P(k)]$ versus $\log _{2}(1 / k)$ series. 3D-PSVG provides the degree of complexity and fractality of the data spaces. Comparison 
among the values of 3D-PSVG calculated for the same 3-dimentional data space taken from 3 perspectives- $(y, z, x),(z, x, y)$ and $(x, y, z)$, is listed in Table 1 . It reveals that the scaling pattern is substantially varied among the 3 perspectives of the same experimental data space. The 3D-PSVG value is lowest and highest for $(x, y, z)$ and $(y, z, x)$ perspectives, respectively. This again proves the rigorousness of the proposed method which is capable of extracting the inherent degree of complexity, self-similarity and fractality from the deepest level of the supersymmetric data. This observation may be accredited to occurrence of some unconventional phenomena like SUSY.

We conclude that the high degree of long-range correlation, fractality and scale-freeness derived from the signatures of final state particles generated in the multi-particle production process from supersymmetric data of $p p$ collisions at $7 \mathrm{TeV}$ of the CMS Collaboration, can be attributed to the dynamics of different kinds of resonance-like states or other extraordinary phenomenon like SUSY inherent in the multi-particle production process in high energy interaction. Further, inspired by the complex-network-based methodology of Visibility Graphs to analyze the long-range correlation, fractality and the inherent scale-freeness of one dimensional data series proposed by Lacasa et al. [66,67], we have proposed the novel, complex-network-based method of the 3D Visibility Graph method which extracts the the inherent degree of scale-freeness embedded at the deepest level in a 3-dimentional space. Thus, the proposed method has the potential to identify prospective SUSY which the traditional methods of analyzing the spectrum of invariant mass and $p_{T}$ may not be able to detect. Moreover, the method is novel, rigorous and simple enough, and hence capable of decoding the dynamics by analyzing the component-space (in the $X, Y$ and $Z$ coordinates) made up of corresponding values of a single variable of $p_{T}$ extracted from 4-momenta of the signatures of final state of the pair of mega-jets produced in the $p p$ collision at $7 \mathrm{TeV}$ from CMS Collaboration [36].

In future explorations, the proposed method can be applied over different high energy interactions to analyze the underlying dynamics manifested in their final state signatures, to the deepest level. This may provide a clue for exploring possible open charm and open beauty decays, other exotic resonance states or other unusual processes in multi-particle production process even from the perspective beyond the SM.

Author Contributions: Conceptualization, S.B. and A.B.; methodology, S.B.; software, S.B.; validation, S.B., and A.B.; formal analysis, A.B.; investigation, A.B.; resources, CERN; data curation, S.B.; writing-original draft preparation, S.B.; writing-review and editing, S.B. All authors have read and agreed to the published version of the manuscript.

Funding: This research received no external funding.

Acknowledgments: We thank CERN, the CMS Collaboration and the CMS Data Preservation and Open Access (DPOA) team for making research-grade collider data available to the public. We specifically thank Kati Lassila-Perini from CMS Collaboration for helping us in setting up the CMS-VM environment for processing CMS Open Data. We also thank Debashis Bandyopadhyay for helping us in language related corrections in the manuscript.

Conflicts of Interest: The authors declare no conflict of interest.

\section{References}

1. Glashow, S.L. Partial-symmetries of weak interactions. Nucl. Phys. 1961, 22, 579-588. [CrossRef]

2. Weinberg, S. A Model of Leptons. Phys. Rev. Lett. 1967, 19, 1264-1266. [CrossRef]

3. Glashow, S.L.; Iliopoulos, J.; Maiani, L. Weak Interactions with Lepton-Hadron Symmetry. Phys. Rev. D 1970, 2, 1285-1292. [CrossRef]

4. Englert, F.; Brout, R. Broken symmetry and the mass of gauge vector mesons. Phys. Rev. Lett. 1964, 13, $321-323$. [CrossRef]

5. Higgs, P.W. Broken symmetries and the masses of gauge bosons. Phys. Rev. Lett. 1964, 13, 508-509. [CrossRef]

6. Guralnik, G.S.; Hagen, C.R.; Kibble, T.W. Global conservation laws and massless particles. Phys. Rev. Lett. 1964, 13, 585-587. [CrossRef] 
7. Aad, G.; et al. [ATLAS Collaboration]. Observation of a new particle in the search for the Standard Model Higgs boson with the ATLAS detector at the LHC. Phys. Lett. Sect. B Nucl. Elem. Part. High-Energy Phys. 2012, 716, 1-29. [CrossRef]

8. Chatrchyan, S.; et al. [CMS Collaboration]. Observation of a new boson at a mass of $125 \mathrm{GeV}$ with the CMS experiment at the LHC. Phys. Lett. Sect. B Nucl. Elem. Part. High-Energy Phys. 2012, 716, 30-61. [CrossRef]

9. Evans, L.; Bryant, P. LHC Machine. J. Instrum. 2008, 3, S08001. [CrossRef]

10. Salam, A.; Sezgin, E.; Gol'fand, Y.A.; Likhtman, E.P. Extension of the algebra of Poincare group generators and violation of P-invariance. In Pisma Zh.Eksp.Teor.Fiz.; World Scientific: Singapore, 1971; Volume 13, pp. 452-455. [CrossRef]

11. Wess, J.; Zumino, B. Supergauge transformations in four dimensions. Nucl. Phys. B 1974, 70, 39-50. [CrossRef]

12. Nilles, H. Supersymmetry, supergravity and particle physics. Phys. Rep. 1984, 110, 1-162. [CrossRef]

13. Haber, H. The search for supersymmetry: Probing physics beyond the standard model. Phys. Rep. 1985, 117, 75-263. [CrossRef]

14. Barbieri, R.; Ferrara, S.; Savoy, C. Gauge models with spontaneously broken local supersymmetry. Phys. Lett. B 1982, 119, 343-347. [CrossRef]

15. Dawson, S.; Eichten, E.; Quigg, C. Search for supersymmetric particles in hadron-hadron collisions. Phys. Rev. D 1985, 31, 1581-1637. [CrossRef] [PubMed]

16. Witten, E. Dynamical breaking of supersymmetry. Nucl. Phys. B 1981, 188, 513-554. [CrossRef]

17. Dimopoulos, S.; Georgi, H. Softly broken supersymmetry and SU(5). Nucl. Phys. B 1981, 193, 150-162. [CrossRef]

18. Jungman, G.; Kamionkowski, M.; Griest, K. Supersymmetric dark matter. Phys. Rep. 1996, 267, 195-373. [CrossRef]

19. Chatrchyan, S.; et al. [CMS Collaboration]. Search for physics beyond the standard model in events with a $Z$ boson, jets, and missing transverse energy in pp collisions at $\sqrt{s}=7$ TeV. Phys. Lett. B 2012, 716, 260-284. [CrossRef]

20. Chatrchyan, S.; et al. [CMS Collaboration]. Search for new physics in events with opposite-sign leptons, jets, and missing transverse energy in pp collisions at $\sqrt{s}=7 \mathrm{TeV}$. Phys. Lett. B 2013, 718, 815-840. [CrossRef]

21. Chatrchyan, S.; et al. [CMS Collaboration]. Search for supersymmetry in events with opposite-sign dileptons and missing transverse energy using an artificial neural network. Phys. Rev. D 2013, 87, 072001. [CrossRef]

22. Golfand, Y.; Likhtman, E. Extension of the Algebra of Poincare Group Generators and Violation of p Invariance. JETP Lett. 1971, 13, 323-326.

23. Ramond, P. Dual Theory for Free Fermions. Phys. Rev. D 1971, 3, 2415-2418. [CrossRef]

24. Volkov, D.V.; Akulov, V.P. Possible universal neutrino interaction. In Supersymmetry Quantum Field Theory; Springer: Berlin/Heidelberg, Germany, 2007; pp. 383-385. [CrossRef]

25. Fayet, P. Supergauge invariant extension of the Higgs mechanism and a model for the electron and its neutrino. Nucl. Phys. B 1975, 90, 104-124. [CrossRef]

26. Abazov, V.E.A. Search for squarks and gluinos in events with jets and missing transverse energy using $2.1 \mathrm{fb}^{-1}$ of $p \bar{p}$ collision data at $\sqrt{s}=1.96$ TeV. Phys. Lett. B 2008, 660, 449-457. [CrossRef]

27. Aaltonen, T.E.A. Inclusive Search for Squark and Gluino Production in $p p$ Collisions at $\sqrt{ } \mathrm{s}=1.96 \mathrm{TeV}$. Phys. Rev. Lett. 2009, 102, 121801. [CrossRef]

28. Aad, G.; et al. [ATLAS Collaboration]. Search for scalar top quark pair production in natural gauge mediated supersymmetry models with the ATLAS detector in $p p$ collisions at $\sqrt{s}=7 \mathrm{TeV}$. Phys. Lett. Sect. B Nucl. Elem. Part High-Energy Phys. 2012, 715, 44-60. [CrossRef]

29. Aad, G.; et al. [ATLAS Collaboration]. Search for supersymmetry in events with three leptons and missing transverse momentum in $\sqrt{s}=7 \mathrm{TeV} p p$ collisions with the ATLAS detector. Phys. Rev. Lett. 2012, 108. [CrossRef]

30. Chatrchyan, S.; et al. [CMS Collaboration]. Search for supersymmetry in hadronic final states using MT2 in $p p$ collisions at $\sqrt{ } s=7 \mathrm{TeV}$. J. High Energy Phys. 2012, 2012. [CrossRef]

31. Farrar, G.R.; Fayet, P. Phenomenology of the production, decay, and detection of new hadronic states associated with supersymmetry. Phys. Lett. B 1978, 76, 575-579. [CrossRef]

32. Aad, G.; et al. [ATLAS Collaboration]. Search for direct production of charginos, neutralinos and sleptons in final states with two leptons and missing transverse momentum in $p p$ collisions at $\sqrt{ } s=8 \mathrm{TeV}$ with the ATLAS detector. J. High Energy Phys. 2014, 2014. [CrossRef]

33. Khachatryan, V; et al. [CMS Collaboration]. Searches for electroweak production of charginos, neutralinos, and sleptons decaying to leptons and W, Z, and Higgs bosons in pp collisions at 8 TeV. Eur. Phys. J. C 2014, 74, 3036. [CrossRef]

34. Chatrchyan, S.; et al. [CMS Collaboration]. Inclusive Search for Supersymmetry Using Razor Variables in $p p$ Collisions at $\sqrt{ } \mathrm{s}=7$ TeV. Phys. Rev. Lett. 2013, 111, 081802. [CrossRef] [PubMed] 
35. Chatrchyan, S.; et al. [CMS Collaboration]. Search for supersymmetry with razor variables in $p p$ collisions at $\sqrt{ } \mathrm{s}=7$ TeV. Phys. Rev. D 2014, 90, 112001. [CrossRef]

36. CMS Collaboration. MultiJet Primary Dataset in AOD Format from RunB of 2010. CERN Open Data Portal. (/Multijet/Run2010B-Apr21ReReco-v1/AOD), 2014. Available online: http:/ /opendata.cern.ch/record/11 (accessed on 20 August 2020). [CrossRef]

37. Alwall, J.; Schuster, P.C.; Toro, N. Simplified models for a first characterization of new physics at the LHC. Phys. Rev. D 2009, 79, 075020. [CrossRef]

38. Alwall, J.; Le, M.P.; Lisanti, M.; Wacker, J.G. Model-independent jets plus missing energy searches. Phys. Rev. D 2009, 79, 015005. [CrossRef]

39. Alves, D.; Arkani-Hamed, N.; Arora, S.; Bai, Y.; Baumgart, M.; Berger, J.; Buckley, M.; Butler, B.; Chang, S.; Cheng, H.-C.; et al. Simplified models for LHC new physics searches. J. Phys. G Nucl. Part. Phys. 2012, 39, 105005. [CrossRef]

40. Bialas, A.; Peschanski, R. Moments of rapidity distributions as a measure of short-range fluctuations in high-energy collisions. Nucl. Phys. Sect. B 1986, 273, 703-718. [CrossRef]

41. Bialas, A.; Peschanski, R. Intermittency in multiparticle production at high energy. Nucl. Phys. Sect. B 1988, 308, 857-867. [CrossRef]

42. De Wolf, E.; Dremin, I.; Kittel, W. Scaling laws for density correlations and fluctuations in multiparticle dynamics. Phys. Rep. 1996, 270, 1-141. [CrossRef]

43. Hwa, R.C. Fractal measures in multiparticle production. Phys. Rev. D 1990, 41, 1456-1462. [CrossRef]

44. Paladin, G.; Vulpiani, A. Anomalous scaling laws in multifractal objects. Phys. Rep. 1987, 156, 147-225. [CrossRef]

45. Grassberger, P.; Procaccia, I. Dimensions and entropies of strange attractors from a fluctuating dynamics approach. Phys. D Nonlinear Phenom. 1984, 13, 34-54. [CrossRef]

46. Halsey, T.C.; Jensen, M.H.; Kadanoff, L.P.; Procaccia, I.; Shraiman, B.I. Fractal measures and their singularities: The characterization of strange sets. Phys. Rev. A 1986, 33, 1141-1151. [CrossRef] [PubMed]

47. Takagi, F. Multifractal structure of multiplicity distributions in particle collisions at high energies. Phys. Rev. Lett. 1994, 72, 32-35. [CrossRef]

48. Peng, C.K.; Buldyrev, S.V.; Havlin, S.; Simons, M.; Stanley, H.E.; Goldberger, A.L. Mosaic organization of DNA nucleotides. Phys. Rev. E 1994, 49, 1685-1689. [CrossRef]

49. Kantelhardt, J.W.; Zschiegner, S.A.; Koscielny-Bunde, E.; Havlin, S.; Bunde, A.; Stanley, H. Multifractal detrended fluctuation analysis of nonstationary time series. Phys. A Stat. Mech. Appl. 2002, 316, 87-114. [CrossRef]

50. Albajar, C.; Allkofer, O.C.; Apsimon, R.; Bartha, S.; Bezaguet, A.; Böhrer, A.; Buschbeck, B.; Cennini, P.; Cittolin, S.; Clayton, E.; et al. Multifractal analysis of minimum bias events in $\sqrt{s}=630 \mathrm{GeV}$ anti-pp collisions. Z. Für Phys. C Part. Fields 1992. [CrossRef]

51. Suleymanov, M.; Sumbera, M.; Zborovsky, I. Entropy and Multifractal Analysis of Multiplicity Distributions from $p p$ Simulated Events up to LHC Energies. 2003. Available online: http:/ /xxx.lanl.gov/abs/hep-ph/0304206 (accessed on 20 August 2020).

52. Zhang, Y.X.; Qian, W.Y.; Yang, C.B. Multifractal structure of pseudorapidity and azimuthal distributions of the shower particles in au + au collisions at $200 \mathrm{~A} \mathrm{GeV.} \mathrm{Int.} \mathrm{J.} \mathrm{Mod.} \mathrm{Phys.} \mathrm{A} \mathrm{2008,} \mathrm{23,} \mathrm{2809-2816.} \mathrm{[CrossRef]}$

53. Ferreiro, E.G.; Pajares, C. High multiplicity $p p$ events and J/ $\psi$ production at energies available at the CERN Large Hadron Collider. Phys. Rev. C Nucl. Phys. 2012, 86. [CrossRef]

54. Tokarev, M.; Dedovich, T.; Zborovský, I. Self-Similarity of Jet and Top-Quark Production at Tevatron and LHC. In Particle Physics at the Year of Centenary of Bruno Pontecorvo; World Scientific: Singapore, 2015; pp. 186-190. [CrossRef]

55. Tokarev, M.; Zborovský, I. Self-similarity of strangeness production in $p p$ collisions at RHIC. J. Phys. Conf. Ser. 2016, 668, 012087. [CrossRef]

56. Tokarev, M.V.; Zborovský, I. Self-similarity of proton spin and asymmetry of jet production. Phys. Part. Nucl. Lett. 2015, 12, 214-220. [CrossRef]

57. Baldina, E.; Baldin, A. Relativistically invariant self-similarity approach for description of collective phenomena. EPJ Web Conf. 2017, 138. [CrossRef]

58. Sonoda, T. Application of Self-Similar Symmetry Model to Dark Energy. Preprints 2018. [CrossRef]

59. Bhaduri, S.; Bhaduri, A.; Ghosh, D. Study of Di-Muon Production Process in $p p$ Collision in CMS Data from Symmetry Scaling Perspective. Adv. High Energy Phys. 2020, 2020, 1-17. [CrossRef]

60. CMS Collaboration(2016). MuOnia Primary Dataset in AOD Format from RunA of 2011. CERN Open Data Portal. (/MuOnia/Run2011A-12Oct2013-v1/AOD), 2016. Available online: http:/ / opendata.cern.ch/record/27 (accessed on 20 August 2020). [CrossRef] 
61. CMS Collaboration(2017). MuOniaParked Primary Dataset in AOD Format from Run of 2012. CERN Open Data Portal. (/MuOniaParked/Run2012B-22Jan2013-v1/AOD), 2017. Available online: http://opendata.cern.ch/record/6017 (accessed on 20 August 2020). [CrossRef]

62. Albert, R.; Barabási, A.L. Statistical mechanics of complex networks. Rev. Mod. Phys. 2002, 74, 47-97. [CrossRef]

63. Barabási, A.L. The network takeover. Nat. Phys. 2011, 8, 14-16. [CrossRef]

64. Havlin, S.; Kenett, D.Y.; Ben-Jacob, E.; Bunde, A.; Cohen, R.; Hermann, H.; Kantelhardt, J.W.; Kertész, J.; Kirkpatrick, S.; Kurths, J.; et al. Challenges in network science: Applications to infrastructures, climate, social systems and economics. Eur. Phys. J. Spec. Top. 2012, 214, 273-293. [CrossRef]

65. Zhao, L.; Li, W.; Cai, X. Structure and dynamics of stock market in times of crisis. Phys. Lett. A 2016, 380, 654-666. [CrossRef]

66. Lacasa, L.; Luque, B.; Ballesteros, F.; Luque, J.; Nuno, J.C. From time series to complex networks: The visibility graph. Proc. Natl. Acad. Sci. USA 2008, 105, 4972-4975. [CrossRef]

67. Lacasa, L.; Luque, B.; Luque, J.; Nuño, J.C. The visibility graph: A new method for estimating the Hurst exponent of fractional Brownian motion. EPL (Europhys. Lett.) 2009, 86, 30001. [CrossRef]

68. Stern, M.A. Ueber eine zahlentheoretische Funktion. J. Für Die Reine Und Angew. Math. 1858, 55, 193-220,

69. Hofstadter, D. Godel, Escher, Bach: An Eternal Golden Braid. New Soc. 1980, 54, 431. [CrossRef]

70. Schroeder, M.; Wiesenfeld, K. Fractals, Chaos, Power Laws: Minutes from an Infinite Paradise; Physics Today: New York, NY, USA, 1991; Volume 44, pp. 91-91. [CrossRef]

71. Hausdorff, J.M.; Purdon, P.L.; Peng, C.K.; Ladin, Z.; Wei, J.Y.; Goldberger, A.L. Fractal dynamics of human gait: Stability of long-range correlations in stride interval fluctuations. J. Appl. Physiol. 1996, 80, 1448-1457. [CrossRef]

72. Goldberger, A.L.; Amaral, L.A.N.; Hausdorff, J.M.; Ivanov, P.C.; Peng, C.K.; Stanley, H.E. Fractal dynamics in physiology: Alterations with disease and aging. Proc. Natl. Acad. Sci. USA 2002, 99, 2466-2472. [CrossRef]

73. Jiang, S.; Bian, C.; Ning, X.; Ma, Q.D.Y. Visibility graph analysis on heartbeat dynamics of meditation training. Appl. Phys. Lett. 2013, 102, 253702. [CrossRef]

74. Bhaduri, S.; Ghosh, D. Multiplicity fluctuation and phase transition in high-energy collision-A chaos-based study with complex network perspective. Int. J. Mod. Phys. A 2016, 31, 1650185. [CrossRef]

75. Bhaduri, S.; Ghosh, D. Pion Fluctuation in High-energy Collisions-A Chaos-based Quantitative Estimation with Visibility Graph Technique. Acta Phys. Pol. B 2017, 48, 741. [CrossRef]

76. Bhaduri, S.; Ghosh, D. Fractal study of pion void probability distribution in ultrarelativistic nuclear collision and its target dependence. Mod. Phys. Lett. A 2016, 31, 1650158. [CrossRef]

77. Bhaduri, A.; Bhaduri, S.; Ghosh, D. Azimuthal pion fluctuation in ultra relativistic nuclear collisions and centrality dependence-A study with chaos based complex network analysis. Phys. Part. Nucl. Lett. 2017, 14, 576-583. [CrossRef]

78. Bhaduri, S.; Bhaduri, A.; Ghosh, D. A new approach of chaos and complex network method to study fluctuation and phase transition in nuclear collision at high energy. Eur. Phys. J. A 2017, 53, 135. [CrossRef]

79. Bhaduri, S.; Bhaduri, A.; Ghosh, D. Clan-Model of Particle Production Process-Revisited in Chaos-based Complex Network Scenario. Phys. Part. Nucl. Lett. 2018, 15, 446-455. [CrossRef]

80. Bhaduri, S.; Bhaduri, A.; Ghosh, D. Study of multiplicity dependence of pion fluctuations in $\pi^{-}-\mathrm{AgBr}$ collisions at $350 \mathrm{GeV}$ using complex network approach. Pramana J. Phys. 2019, 92, 4. [CrossRef]

81. Bhaduri, S.; Ghosh, D. Azimuthal Anisotropy in High-Energy Nuclear Collision: An Approach Based on Complex Network Analysis. Adv. High Energy Phys. 2018, 2018, 1-9. [CrossRef]

82. Bhaduri, S.; Bhaduri, A.; Ghosh, D. Pion Fluctuation Study in Pb-Pb Collision at $2.76 \mathrm{TeV}$ per Nucleon Pair from ALICE Experiment with Chaos and Complex Network-Based Methods. Phys. Part. Nucl. Lett. 2019, 16, 229-239. [CrossRef]

83. Bhaduri, S.; Bhaduri, A.; Ghosh, D. Symmetry-Scaling Based Complex Network Approach to Explore Exotic Hadronic States in High-Energy Collision. Phys. Part. Nucl. Lett. 2019, 16, 779-788. [CrossRef]

84. ALICE Collaboration. Pb-Pb data sample at the collision energy of $2.76 \mathrm{TeV}$ per nucleon pair from run number 139038. CERN Open Data Portal. [CrossRef]

85. Werner, K.; Knospe, A.G.; Markert, C.; Guiot, B.; Karpenko, I.; Pierog, T.; Sophys, G.; Stefaniak, M.; Bleicher, M.; Steinheimer, J. Resonance production in high energy collisions from small to big systems. EPJ Web Conf. 2018, 171, 09002. [CrossRef]

86. Ahmadlou, M.; Adeli, H.; Adeli, A. Improved visibility graph fractality with application for the diagnosis of Autism Spectrum Disorder. Phys. A Stat. Mech. Appl. 2012, 391, 4720-4726. [CrossRef] 
87. CMS Collaboration; CMS List of Validated Runs for Primary Datasets of 2010 Data Taking. Available online: http:/ / opendata.cern.ch/record/1000 (accessed on 20 August 2020). [CrossRef]

88. CernVM Appliance. CernVM Software Appliance. Available online: https://cernvm.cern.ch (accessed on 20 August 2020)

89. CernVM Appliance. CMS VM Image, for 2010 CMS Open Data. Available online: http:/ / opendata.cern.ch/ record/250 (accessed on 20 August 2020).

90. Duarte, J. Razor Filter and Analyzer for SUSY Searches. Available online: http://opendata.cern.ch/record/ 553 (accessed on 20 August 2020). [CrossRef]

91. Clauset, A.; Shalizi, C.R.; Newman, M.E.J. Power-Law Distributions in Empirical Data. SIAM Rev. 2009, 51, 661-703. [CrossRef]

(C) 2020 by the authors. Licensee MDPI, Basel, Switzerland. This article is an open access article distributed under the terms and conditions of the Creative Commons Attribution (CC BY) license (http:/ / creativecommons.org/licenses/by/4.0/). 